University of Warwick institutional repository: http://go.warwick.ac.uk/wrap This paper is made available online in accordance with publisher policies. Please scroll down to view the document itself. Please refer to the repository record for this item and our policy information available from the repository home page for further information.

To see the final version of this paper please visit the publisher's website. Access to the published version may require a subscription.

Author(s): A. A. PINTO and D. A. RAND

Article Title: Existence uniqueness and ratio decomposition for Gibbs states via duality

Year of publication: 2001

Link to published version:

http://dx.doi.org/10.1017/S0143385701001262

Publisher statement: None 


\title{
Existence, uniqueness and ratio decomposition for Gibbs states via duality
}

\author{
A. A. PINTO $\dagger$ and D. A. RAND $\ddagger$ \\ $\dagger$ Departamento de Matemática Aplicada, Faculdade de Ciências, Universidade do Porto, \\ 4000 Porto, Portugal \\ (e-mail: aapinto@fc.up.pt) \\ $\ddagger$ Nonlinear Systems Laboratory, Mathematics Institute, University of Warwick, \\ Coventry CV4 7AL, UK \\ (e-mail:dar@maths.warwick.ac.uk)
}

(Received 20 November 1998 and accepted in revised form 4 April 2000)

\begin{abstract}
We give an elementary proof of existence and uniqueness of Gibbs states for Hölder weight systems on subshifts of finite type. This uses a notion of duality for such subshifts. The approach of Paterson [2] is used to construct a measure with a prescribed Jacobian and the duality is used to produce an invariant measure from this
\end{abstract}

\section{Introduction}

In this paper we give a novel and elementary proof of the existence and uniqueness of Gibbs states for Hölder weight systems. A bonus of this approach is that it leads directly to a decomposition of the measure as an integral of an explicitly given canonical ratio function with respect to a measure which is dual to the Gibbs state. See the corollary to Theorem 1 for the precise statement and Definition 3 for the definition of the canonical ratio function which corresponds to the conditional measures along backward paths.

The novelty of our approach is to use a notion of duality and to combine it with the approach to constructing measures pioneered by Paterson [2] in the context of the limit sets of Fuchsian groups and used by Sullivan [4] to construct conformal measures for Julia sets. This duality plays the central role in our approach through the following observations: (i) given a Hölder function $J$ on the shift space $\Sigma$ (or, equivalently, a Hölder weight system) there is a unique measure $v$ on $\Sigma$ whose Jacobian is $J$; (ii) the weight system determined by $v$ determines a unique measure $\mu^{*}$ on the dual space to $\Sigma$ which is invariant under the dual mapping $f_{*}$ on $\Sigma^{*}$; and (iii) the measure $\mu$ dual to $\mu^{*}$ is the required shift-invariant Gibbs state for $J$ and its Jacobian is explicitly given by our construction because it is the reciprocal of the scaling function of $\mu^{*}$. The Jacobian of the measure $\mu^{*}$ provides a nice characterization of the Gibbs state and we deduce that there is a natural one-to-one 
correspondence between the Hölder Gibbs states on $\Sigma$ and the Hölder functions $\sigma(\xi)$ on $\Sigma^{*}$ which satisfy the simple matching condition given below in Definition 2 .

The ratio decomposition is particularly useful in certain situations and can be used to link certain Gibbs states with smooth structures. For example, in [3] it is used to construct all $C^{1+}$ Anosov diffeomorphisms and hyperbolic attractors on surfaces that have an invariant measure that is absolutely continuous with respect to the two-dimensional Lebesgue measure (in the case of Anosov systems) or the Hausdorff measure of the attractor. In particular, for such systems, it allows us to introduce a notion of duality between the induced affine structures on the stable and unstable foliations. For the Anosov systems the SRB measure for the system is absolutely continuous if and only if one of these structures is the dual of the other.

\section{Gibbs states and statement of main theorem}

Let us recall the definition of a one-sided subshift of finite type $\Sigma=\Sigma_{A}$. The elements of $\Sigma$ are all the infinite right-handed words $w=w_{0} w_{1} \ldots$ in the symbols $1, \ldots, k$ such that for all $i \geq 0, A_{w_{i} w_{i+1}}=1$. Here $A=\left(A_{i j}\right)$ is any matrix with entries 0 and 1 such that $A^{n}$ has all entries positive for some $n \geq 1$. We write $w \stackrel{n}{\sim} w^{\prime}$ if the two words $w, w^{\prime} \in \Sigma$ agree on their first $n$ entries. The metric $d$ on $\Sigma$ is given by $d\left(w, w^{\prime}\right)=2^{-n}$ if $n \geq 0$ is the largest such that $w \stackrel{n}{\sim} w^{\prime}$. Together with this metric $\Sigma$ is a compact metric space. The shift $f: \Sigma \rightarrow \Sigma$ is the mapping which sends $w_{0} w_{1} \ldots$ to $w_{1} w_{2} \ldots$ It is a local homeomorphism.

An $n$-cylinder $\Sigma_{w}, w \in \Sigma_{n}$, consists of all those words $w^{\prime}$ in $\Sigma$ such that $\stackrel{n}{\sim} w^{\prime}$. If $C$ is an $n$-cylinder then we define $m C$ to be the $(n-1)$-cylinder containing $C$ and denote by $n(C)$ the depth $n$ of $C$. A 1-cylinder is also called a primary cylinder.

Together with $\Sigma$ we will consider the augmented space $\Theta$ which consists of both the infinite right-handed words in $\Sigma$ and their finite subwords. Let $\Theta_{\text {fin }}$ denote the subset of finite words. Then we can identify $\Theta_{\text {fin }}$ with the set of cylinders in $\Sigma$ via the association $w \leftrightarrow \Sigma_{w}$. This set has two natural oriented tree structures:

(a) $\Theta_{\text {fin }}^{m}$ in which all the oriented edges connect a cylinder $C$ to $m C$; and

(b) $\Theta_{\text {fin }}^{f}$ in which all the oriented edges are from the cylinder $C$ to $f C$.

An admissible backward path in either of these trees is a finite or infinite sequence $\left\{C_{j}\right\}$ of cylinders indexed by either $j=0, \ldots, n$ or $j=0,1, \ldots$ and such that $C_{0}$ is a primary cylinder and such that there is an oriented edge from $C_{j}$ to $C_{j-1}$ for all $j>0$. Clearly the infinite paths in $\Theta_{\text {fin }}^{m}$ correspond to points of $\Sigma$.

Definition 1. The dual $\Sigma^{*}$ of $\Sigma$ is the set of all infinite admissible backward paths in $\Theta_{\text {fin }}^{f}$ together with the metric defined as follows: $d^{*}\left(\left\{C_{j}\right\},\left\{C_{j}^{\prime}\right\}\right)=2^{-n}$ if $C_{j}=C_{j}^{\prime}$ for $0<j<n$ and $C_{n} \neq C_{n}^{\prime}$. The dual $\Theta^{*}$ of $\Theta$ is defined similarly except that both finite and infinite admissible backward paths in $\Theta_{\text {fin }}^{f}$ are used.

Note that one can identify the elements of $\Sigma^{*}$ with those infinite left-handed words $\ldots w_{1} w_{0}$ in the symbols $1, \ldots, k$ such that $A_{w_{i} w_{i-1}}=1$.

We note that for both $\Sigma$ and $\Sigma^{*}$ a cylinder is given by prescribing a finite admissible backward path $\left\{C_{j}\right\}_{j=0}^{n-1}$ (respectively in $\Theta_{\text {fin }}^{m}$ and in $\Theta_{\text {fin }}^{f}$ ), and it is then equal to the set 
of all infinite admissible backward paths $\left\{D_{j}\right\}$ such that $D_{j}=C_{j}$ for $0<j<n$. Since this finite path is determined by $C_{n-1}$ there is a one-to-one correspondence between the cylinders of $\Sigma$ and $\Sigma^{*}$. Specifically, this is given as follows: if $C$ is an $n$-cylinder of $\Sigma$ then the cylinder $C^{*}$ of $\Sigma^{*}$ consists of all infinite admissible backward paths $\left\{C_{j}\right\}_{j=0}^{\infty}$ in $\Theta_{\text {fin }}^{f}$ such that $C_{n-1}=C$. We also define duals to $m$ and $f$ : if $C^{*}=\left\{C_{j}\right\}_{j=0}^{n-1}$ is an $n$ cylinder of $\Sigma^{*}, m_{*} C^{*}$ is the $(n-1)$-cylinder $\left\{f C_{j}\right\}_{j=1}^{n-1}$ of $\Sigma^{*}$ containing $C^{*}$ and $f_{*} C^{*}$ is the $(n-1)$-cylinder $\left\{m C_{j}\right\}_{j=1}^{n-1}$. Note how these translate under duality:

$$
m_{*} C^{*}=(f C)^{*} \quad \text { and } \quad f_{*} C^{*}=(m C)^{*} .
$$

2.1. Notation. Throughout, if $\phi(n)$ is some quantity depending upon $n \in \mathbb{N}$ and $L \in \mathbb{R}$, we use the notation $\phi(n) \in\left(1 \pm \mathcal{O}\left(v^{n}\right)\right) L$ to mean that there exists a constant $c>0$ depending only upon explicitly mentioned quantities such that for all $n \geq 0$, $1-c v^{n}<\phi(n) / L<1+c v^{n}$.

2.2. The potential. Now consider a function $\ell$ defined on $\Theta_{\text {fin }}$ and with the following properties: there exists $0<\omega<\omega^{\prime}<1$ such that if $C$ is an $n$-cylinder then

$$
\mathcal{O}\left(\omega^{n}\right)<\ell(C)<\mathcal{O}\left(\omega^{\prime n}\right)
$$

and there exists $0<v<1$ such that the following two equivalent conditions hold:

(i) if $C$ is an $n$-cylinder with $n>0$ then $\sigma_{\ell}(C)=\ell(C) / \ell(m C)$ converges exponentially along backward orbits, i.e. $\sigma_{\ell}(C) \in\left(1 \pm \mathcal{O}\left(\nu^{n}\right)\right) \sigma_{\ell}(f C)$;

(ii) if $C$ is an $n$-cylinder with $n>0$ then $J_{\ell}(C)=\ell(f C) / \ell(C)$ converges exponentially along nested sequences, i.e. $J_{\ell}(C) \in\left(1 \pm \mathcal{O}\left(v^{n}\right)\right) J_{\ell}(m C)$.

We leave the proof of the equivalence to the reader, but note that it comes from the relation

$$
\frac{\sigma_{\ell}(f C)}{\sigma_{\ell}(C)}=\frac{J_{\ell}(C)}{J_{\ell}(m C)} .
$$

It also follows from these conditions that the limits defining the following functions $\sigma_{\ell}$ and $J_{\ell}$ are reached exponentially fast and that consequently these functions are Hölder continuous: if $\xi=\left\{C_{n}\right\}_{n=0}^{\infty} \in \Sigma^{*}$ where $C_{n}$ is an $n$-cylinder and $f C_{n+1}=C_{n}$ and if $x=\bigcap_{n \geq 0} D_{n}$ where $D_{n}$ is a $n$-cylinder with $m D_{n+1}=D_{n}$ then

$$
\sigma_{\ell}(\xi)=\lim _{n \rightarrow \infty} \sigma_{\ell}\left(C_{n}\right) \quad \text { and } \quad J_{\ell}(x)=\lim _{n \rightarrow \infty} J_{\ell}\left(D_{n}\right) .
$$

Definition 2. Such a system of weights $\ell$ is called a Hölder weight function. We call $\sigma_{\ell}$ the scaling function of $\ell$ and $J_{\ell}$ the Jacobian. The Hölder weight function is said to satisfy the matching condition or to match if for all $\xi \in \Sigma^{*}$,

$$
\sum_{f_{*} \xi^{\prime}=\xi} \sigma_{\ell}\left(\xi^{\prime}\right)=1 .
$$

The matching condition is equivalent to the following: there is $0<\theta<1$ such that for all $n \geq 0$ and all $n$-cylinders $C, \sum \sigma_{\ell}\left(C^{\prime}\right)=1 \pm \mathcal{O}\left(\theta^{n}\right)$ (sum over $(n+1)$-cylinders $C^{\prime}$ contained in $C$ ). 
Consider the sums $Z_{s}^{n}=\sum_{C} \ell(C) e^{-s n}$ where the sum is over all $n$-cylinders $C$. From (2), for $s>0$ sufficiently large $Z_{s}^{n}$ is bounded away from infinity uniformly in $n \geq 0$. On the other hand, if $s$ is sufficiently negative then $Z_{s}^{n}$ diverges to $\infty$ as $n \rightarrow \infty$. Since if this divergence occurs for a particular value of $s$ then it occurs for all smaller values, there is a critical value $P$ given by $P=\inf \left\{s: Z_{s}^{n}\right.$ uniformly bounded in $\left.n\right\}$. This is called the pressure of $\ell$. It corresponds to the usual definition [1].

2.3. The ratio structure. Before proceeding we need to introduce some notation. Consider a cylinder $C$ in $\Sigma$ and let $C_{1}$ denote the primary cylinder containing $C$. If $C_{n}$ is an $n$-cylinder such that $f^{n-1} C_{n}=C_{1}$ then by $C\left(C_{n}\right)$ we denote $\left(\left.f^{n-1}\right|_{C_{n}}\right)^{-1}(C)$.

Definition 3. Let $\ell$ be a Hölder weight system.

(i) We define the ratio $r_{\ell}(C: D)$ between two cylinders $C$ and $D$ by

$$
r_{\ell}(C: D)=\lim _{s \searrow P} \frac{\sum_{C^{\prime} \subset C} \ell\left(C^{\prime}\right) e^{-n\left(C^{\prime}\right) s}}{\sum_{D^{\prime} \subset D} \ell\left(D^{\prime}\right) e^{-n\left(D^{\prime}\right) s}}
$$

where the sums are respectively over all cylinders contained in or equal to $C$ and $D$. For $s>P$ both numerator and denominator are finite and positive. As part of the proof of the following theorem we will show that the limit as $s \searrow P$ is finite and positive.

(ii) If $\xi=\left(\xi_{n}\right) \in \Sigma^{*}$ let $\sigma(\xi)=\lim _{n \rightarrow \infty} r_{\ell}\left(\xi_{n}: m \xi_{n}\right)$.

(iii) If $\xi \in \Sigma^{*}$ and $C$ is contained in the primary cylinder $\xi_{0}$ then define $r_{\ell, \xi}(C)=$ $\lim _{n \rightarrow \infty} r_{\ell}\left(C\left(\xi_{n}\right): \xi_{n}\right)$.

The limits in (i), (ii) and (iii) exist and are finite and positive (use (9) and (10) to deduce (i), and use the fact that $\ell_{v}(C)=v(C)$ form a matching Hölder weight system to deduce (ii) and (iii) where $v$ is the probability measure constructed in Theorem 1). From (9), (10) and (11), we also get bounds for $r_{\ell}(C: D)$ as presented in the following remark.

Remark 1. Suppose that $C$ is an $m$-cylinder contained in the $n$-cylinder $D$, then

$$
r_{\ell}(C: D)=\mathcal{O}\left(e^{-(m-n) P} \ell(C) / \ell(D)\right) .
$$

If $\ell$ satisfies the matching condition then $P=0$ and for some $0<\theta<1$,

$$
r_{\ell}(C: D) \in\left(1 \pm \mathcal{O}\left(\theta^{n}\right)\right) \frac{\ell(C)}{\ell(D)}
$$

whenever $C$ and $D$ are contained in a common $n$-cylinder. Therefore, for all $\xi=\left\{\xi_{n}\right\}_{n=0}^{\infty} \in$ $\Sigma^{*}$,

$$
\sigma(\xi)=\sigma_{\ell}(\xi)=\lim _{n \rightarrow \infty} \ell\left(\xi_{n}\right) / \ell\left(m \xi_{n}\right) \text { and } r_{\ell, \xi}(C)=\lim _{n \rightarrow \infty} \ell\left(C\left(\xi_{n}\right)\right) / \ell\left(\xi_{n}\right) .
$$

Clearly in these cases the limits are reached exponentially fast and $\sigma_{\ell}(\xi)$ and $r_{\ell, \xi}(C)$ are Hölder in $\xi$. 


\subsection{The Gibbs measure and its dual.}

Definition 4. Suppose that $\mu$ is a $f$-invariant probability measure on $\Sigma$ and $\nu$ a $f_{*}$-invariant probability measure on $\Sigma^{*}$. Then the duals $\mu^{*}$ and $\nu^{*}$ respectively to $\mu$ and $\nu$ are the probability measures defined on $\Sigma^{*}$ and $\Sigma$ by $\mu^{*}\left(C^{*}\right)=\mu(C)$ and $\nu^{*}\left(C^{*}\right)=v(C)$.

Remark 2. In the above definition we use the fact that $\mu^{*}$ is a probability measure (respectively $f_{*}$-invariant) if and only if $\mu$ is $f$-invariant (respectively a probability measure). Similarly for $v$. This is because $f C=D$ (respectively $f_{*} C^{*}=D^{*}$ ) if and only if $m_{*} C^{*}=D^{*}$ (respectively $m C=D$ ).

THEOREM 1. There exist a unique pair of Borel probability measures $v$ on $\Sigma$ and $v^{*}$ on $\Sigma^{*}$ with the following property for some $0<\theta<1$ : if $C$ is an $n$-cylinder of $\Sigma$,

$$
\frac{\nu(f C)}{v(C)} \in\left(1 \pm \mathcal{O}\left(v^{n}\right)\right) J_{l}(C) e^{P}, \quad \frac{\nu^{*}\left(f_{*} C^{*}\right)}{v^{*}\left(C^{*}\right)} \in\left(1 \pm \mathcal{O}\left(\theta^{n}\right)\right) \sigma_{\ell}^{-1}\left(C^{*}\right) e^{P}
$$

and, if $C$ and $D$ are two cylinders, then $v(C) / v(D)=r_{\ell}(C: D)$. Moreover, the weights $\ell_{v}(C)=v(C)$ form a matching Hölder weight system and $\sigma_{\ell_{v}}=\sigma$.

If the weight function $\ell$ satisfies the matching condition then $v^{*}$ is $f_{*}$-invariant and its dual measure $\mu$ satisfies the following equivalent conditions:

(i) if $C$ and $D$ are two cylinders contained in the same $n$-cylinder then $\mu(D) / \mu(C) \in$ $\left(1 \pm \mathcal{O}\left(\theta^{n}\right)\right) \ell(D) / \ell(C)$;

(ii) if $C$ is an $n$-cylinder and $\xi=\left(\xi_{i}\right) \in \Sigma^{*}$ has $\xi_{n}=C$ then

$$
\mu(C) / \mu(m C) \in\left(1 \pm \mathcal{O}\left(\theta^{n}\right)\right) \sigma_{\ell}(\xi) ;
$$

(iii) (ratio decomposition) if $C$ is an $n$-cylinder and $C_{0}$ is the primary cylinder containing C then

$$
\mu(C)=\int_{C_{0}^{*}} r_{\ell, \xi}(C) \mu^{*}(d \xi) .
$$

Here $\mu^{*}$ is the dual measure to $\mu$.

Moreover, for each of the conditions (i), (ii) and (iii), $\mu$ is the unique measure with the given property.

If $J_{\mu}$ is the Jacobian $d(\mu \circ f) / d \mu$ and $x=\bigcap_{n \geq 0} C_{n} \in \Sigma$ where $C_{n}$ is an $n$ cylinder with $m C_{n+1}=C_{n}$ then $J_{\mu}(x)=\lim _{n \rightarrow \infty} v^{*}\left(m_{*} C_{n}^{*}\right) / \nu^{*}\left(C_{n}^{*}\right)$. The Jacobian $J_{v^{*}}(\xi)=d\left(\nu^{*} \circ f\right) / d \nu^{*}(\xi)$ is $\sigma_{\ell}^{-1}(\xi)$.

Remark 3. As part of the proof of the theorem we will prove that, if the Hölder weight system $\ell$ matches and if $\mu$ is any $f$-invariant probability measure satisfying the ratio decomposition (iii), then for all cylinders $C$ of $\Sigma$,

$$
\sum r_{\ell, \xi_{D}}(C) \mu^{*}\left(D^{*}\right) \in\left(1 \pm \mathcal{O}\left(v^{n}\right)\right) \mu(C)
$$

where the sum is over all $n$-cylinders $D$ such that $C \subset f^{n-1} D$, and for each $D$, $\xi_{D}=\left\{\xi_{j}\right\}_{j=0}^{\infty}$ is an infinite backward path with the property that $\xi_{n}=D$.

COROLlary 1. (Existence and uniqueness of Gibbs states) There exist a unique pair of Borel probability measures $\mu$ on $\Sigma$ and $\mu^{*}$ on $\Sigma^{*}$ with the following properties for some $0<\theta<1$ : 
(i) $\quad \mu$ and $\mu^{*}$ are dual to each other and respectively $f$-invariant and $f_{*}$-invariant;

(ii) if $C$ and $D$ are two cylinders contained in the same $n$-cylinder then

$$
\mu(C) / \mu(D) \in\left(1 \pm \mathcal{O}\left(\theta^{n}\right)\right) r_{\ell}(C: D)
$$

(iii) (ratio decomposition) if $C$ is an $n$-cylinder and $C_{0}$ is the primary cylinder containing C then

$$
\mu(C)=\int_{C_{0}^{*}} r_{\ell, \xi}(C) \mu^{*}(d x) .
$$

Either of the conditions (ii) and (iii) characterize the measure $\mu$, i.e. it is the unique measure with the given property.

If $J_{\mu}$ is the Jacobiand $(\mu \circ f) / d \mu$ and $x=\bigcap_{n>0} C_{n} \in \Sigma$ where $C_{n}$ is an n-cylinder with $m C_{n+1}=C_{n}$ then $J_{\mu}(x)=\lim _{n \rightarrow \infty} \mu^{*}\left(m_{*} C_{n}^{*}\right) / \mu^{*}\left(C_{n}^{*}\right)$. Finally, $d\left(\mu^{*} \circ f_{*}\right) / d \mu^{*}=\sigma^{-1}$.

The measure $\mu$ is the Gibbs state for the potential $J_{\ell}$ in the sense of [1], i.e. it is the unique $f$-invariant probability measure which for all cylinders $C$ the ratios $\mu(C) / \ell(C) e^{-n(C) P}$ are uniformly bounded away from 0 and $\infty$.

Remark 4. Note that the ratios $r_{\ell, \xi}$ and $r_{\xi}$ can be different if the weights do not match, and the logarithmic scaling functions $\log \sigma_{\ell}$ and $\log \sigma$ differ at most by a coboundary, i.e. there is a Hölder continuous function $u: \Sigma^{*} \rightarrow \mathbb{R}$ such that $\log \left(\sigma_{\ell}(\xi)\right)=\log (\sigma(\xi))+u\left(f_{*} \xi\right)-$ $u(\xi)$. However, if the weight system $\ell$ matches then $r_{\ell, \xi}=r_{\xi}$ and $\sigma_{\ell}=\sigma$.

COROLlary 2. (Moduli space for Gibbs states) The correspondence between $\sigma$ and $\mu$ given in Corollary 1 gives a natural one-to-one correspondence between Hölder Gibbs states and Hölder scaling functions on the dual space $\Sigma^{*}$ which satisfy the matching condition (3).

\section{Proof of Theorem 1 and Corollary 1}

Consider a Hölder weight system $\ell$ with pressure $P$. We omit the proof of the following lemma because it closely follows that of [2, Lemma 3.1].

LEMMA 1. There is a positive decreasing continuous function $k$ on $[0, \infty]$ with the following properties:

(i) the sums $Z_{s}=\sum_{C} k(\ell(C)) \ell(C) e^{-n(C) s}$ (sum over all cylinders $C$ ) converge for $s>P$ and diverge for $s=P$; and

(ii) for all $\varepsilon>0$ there is a $y_{0}(\varepsilon)>0$ such that $\lambda^{-\varepsilon} \leq k(\lambda y) / k(y) \leq 1$ whenever $\lambda>1$ and $0<\lambda y<y_{0}(\varepsilon)$.

Proof of Theorem 1. First, consider the sum $Z_{s}=\sum_{C} k(\ell(C)) \ell(C) e^{-s n(C)}$ where the sum is over all cylinders $C$ and $k$ is the function given by Lemma 1 . As we have seen above, $Z_{s}<\infty$ for $s>P$ and $Z_{s}$ diverges if $s=P$. We denote $k(\ell(C)) \ell(C)$ by $\tilde{\ell}(C)$ and $\tilde{\ell}(C) e^{-s n(C)}$ by $\tilde{\ell}_{S}(C)$.

Note that the condition Lemma 1(ii) on $k$ and the fact that $\ell(f C)=J_{\ell}(C) \cdot \ell(C)$ implies that for all $\varepsilon>0$, if $J_{\ell}(C) \geq 1$ then $J_{\ell}(C)^{-\varepsilon} \leq k(\ell(f C)) / k(\ell(C)) \leq 1$ and if $J_{\ell}(C)<1$ then $1 \leq k(\ell(f C)) / k(\ell(C)) \leq J_{\ell}(C)^{-\varepsilon}$ provided $\max \{\ell(C), \ell(f C)\}<y_{0}(\varepsilon)$. Since 
$J_{\ell}(C)$ is bounded away from 0 and $\infty$ uniformly in $C$ we deduce that for all $\varepsilon>0$,

$$
\frac{\tilde{\ell}(f C)}{\tilde{\ell}(C)} \in(1 \pm \varepsilon) J_{\ell}(C)
$$

provided $\ell(C)$ is sufficiently small. Similarly, one deduces that

$$
\frac{\tilde{\ell}(m C)}{\tilde{\ell}(C)} \in(1 \pm \varepsilon) \sigma_{\ell}(C)^{-1}
$$

provided $\ell(C)$ is sufficiently small.

For $s>P$ let $v_{s}$ and $v_{s}^{*}$ be the probability measures on $\Theta$ and $\Theta^{*}$ defined by $v_{s}=Z_{s}^{-1} \sum_{x \in \Theta_{\mathrm{fin}}} \tilde{\ell}_{s}(C) \delta_{x}$ and $v_{s}^{*}=Z_{s}^{-1} \sum_{\xi \in \Theta_{\mathrm{fin}}^{*}} \tilde{\ell}_{s}(C) \delta_{\xi}$ where $\delta_{x}$ and $\delta_{\xi}$ are, respectively, the Dirac measures at $x$ and $\xi$.

Now since $\Theta$ and $\Theta^{*}$ are compact metric spaces there exist sequences $s_{i}>0$ and $s_{i}^{*}>0$ converging to $\mathrm{P}$ as $i \rightarrow \infty$ so that the sequence $v_{s_{i}}$ (respectively $v_{s_{i}^{*}}^{*}$ ) converges weakly to a Borel probability measure $v$ on $\Theta$ (respectively $v^{*}$ on $\Theta^{*}$ ). Since $Z_{s_{i}}$ and $Z_{s_{i}^{*}}$ diverge as $i \rightarrow \infty, v$ and $v^{*}$ are respectively concentrated on $\Sigma$ and $\Sigma^{*}$. Thus $v$ and $v^{*}$, respectively, define measures on $\Sigma$ and $\Sigma^{*}$ which we also denote by $v$ and $v^{*}$.

If $w$ is a finite word, consider the cylinder $\Sigma_{w}$ in $\Sigma$ and also the subset $\Theta_{w}$ in $\Theta^{*}$ consisting of all finite and infinite right-handed words agreeing with $w$. We have

$$
v\left(\Sigma_{w}\right)=v\left(\Theta_{w}\right) \approx v_{s_{i}}\left(\Theta_{w}\right)=Z_{s_{i}}^{-1} \sum_{C \subset \Sigma_{w}} \tilde{\ell}_{s_{i}}(C)
$$

where the sum is over all cylinders $C$ contained in $\Sigma_{w}$ and with the approximation converging as $i \rightarrow \infty$. Therefore, by (6), for $\varepsilon>0$

$$
\frac{\nu\left(f \Sigma_{w}\right)}{\nu\left(\Sigma_{w}\right)}=\lim _{i \rightarrow \infty} \frac{\sum_{D \subset f \Sigma_{w}} \tilde{\ell}_{s_{i}}(D)}{\sum_{C \subset \Sigma_{w}} \tilde{\ell}_{s_{i}}(C)}=\lim _{i \rightarrow \infty} \frac{\sum_{C \subset \Sigma_{w}} \tilde{\ell}_{s_{i}}(f C)}{\sum_{C \subset \Sigma_{w}} \tilde{\ell}_{s_{i}}(C)} \in(1 \pm \epsilon) J_{\ell}\left(\Sigma_{w}\right) e^{P}
$$

provided that $\ell\left(\Sigma_{w}\right)$ is sufficiently small. This implies that the Jacobian of $v$ at $x \in \cap_{j=0}^{\infty} C_{n}$ is $J_{v}(x)=d(v \circ f) / d v=\lim _{n \rightarrow \infty} J_{\ell}\left(C_{n}\right) e^{P}$. Since this is Hölder continuous, we obtain that if $\Sigma_{w}$ is an $n$-cylinder then

$$
\frac{v\left(f \Sigma_{w}\right)}{v\left(\Sigma_{w}\right)} \in\left(1 \pm \mathcal{O}\left(\theta^{n}\right)\right) J_{\ell}\left(\Sigma_{w}\right) e^{P}
$$

for some $0<\theta<1$. Thus, the weights $\ell_{v}\left(\Sigma_{w}\right)=v\left(\Sigma_{w}\right)$ form a Hölder weight system.

If $w$ is a word consider the cylinder $\Sigma_{w}^{*}$ in $\Sigma^{*}$ and also the subset $\Theta_{w}^{*}$ in $\Theta^{*}$ consisting of all admissible backward finite and infinite paths agreeing with $w$. We have

$$
v^{*}\left(\Sigma_{w}^{*}\right)=v^{*}\left(\Theta_{w}^{*}\right) \approx v_{s_{i}^{*}}^{*}\left(\Theta_{w}^{*}\right)=Z_{s_{i}^{*}}^{-1} \sum_{C^{*} \subseteq \Sigma_{w}^{*}} \tilde{\ell}_{s_{i}^{*}}(C)=Z_{s_{i}^{*}}^{-1} \sum_{C \rightarrow \Sigma_{w}} \tilde{\ell}_{s_{i}^{*}}(C)
$$

where $C \rightarrow \Sigma_{w}$ means that $f^{k} C=\Sigma_{w}$ for some $k \geq 0$ with the approximation marked $\approx$ converging as $i \rightarrow \infty$. The first sum in this equation is over all cylinders $C^{*}$ contained in or equal to $\Sigma_{w}^{*}$ and the second equals this because by duality (1), $C^{*} \subseteq \Sigma_{w}^{*}$ if and only if 
$f^{k} C=\Sigma_{w}$. Therefore, by construction of $\sigma_{\ell}$, we have that for all $\varepsilon>0$,

$$
\begin{aligned}
\frac{v^{*}\left(f_{*} \Sigma_{w}^{*}\right)}{v^{*}\left(\Sigma_{w}^{*}\right)} & =\lim _{i \rightarrow \infty} \frac{\sum_{f^{k} C=m \Sigma_{w}} \tilde{\ell}_{s_{i}^{*}}(C)}{\sum_{f^{k} C=\Sigma_{w}} \tilde{\ell}_{s_{i}^{*}}(C)} \\
& =\lim _{i \rightarrow \infty} \frac{\sum_{f^{k} C=\Sigma_{w}} \tilde{\ell}_{s_{i}^{*}}(m C)}{\sum_{f^{k} C=\Sigma_{w}} \tilde{\ell}_{s_{i}^{*}}(C)} \in(1 \pm \varepsilon) \sigma_{\ell}\left(\Sigma_{w}\right)^{-1} e^{P},
\end{aligned}
$$

provided that $\ell\left(\Sigma_{w}\right)$ is sufficiently small. This implies that the Jacobian of $v^{*}$ is $J_{v^{*}}(\xi)=$ $d\left(\nu^{*} \circ f_{*}\right) / d v^{*}=\sigma_{\ell}(\xi)^{-1} e^{P}$. Since this is Hölder continuous, we obtain that the weights $\ell_{*}\left(\Sigma_{w}^{*}\right)=v^{*}\left(\Sigma_{w}^{*}\right)$ form a Hölder weight system and indeed, if $\Sigma_{w}$ is an $n$-cylinder,

$$
\frac{\nu^{*}\left(f_{*}\left(\Sigma_{w}^{*}\right)\right)}{v^{*}\left(\Sigma_{w}^{*}\right)} \in\left(1 \pm \mathcal{O}\left(\theta^{n}\right)\right) \sigma_{\ell}\left(\Sigma_{w}\right)^{-1} e^{P}
$$

for some $0<\theta<1$.

Now we consider the uniqueness of $v$ and $v^{*}$. Suppose that $v^{\prime}$ is another measure satisfying (7). Then, if $C$ is an $n$-cylinder,

$$
\frac{v^{\prime}(C)}{v(C)}=\frac{v^{\prime}(C)}{v^{\prime}(f C)} \cdot \frac{v^{\prime}(f C)}{v(f C)} \cdot \frac{v(f C)}{v(C)} \in\left(1 \pm \mathcal{O}\left(\theta^{n}\right)\right) \frac{v^{\prime}(f C)}{v(f C)}
$$

because $v^{\prime}(f C) / v^{\prime}(C)=\left(1 \pm \mathcal{O}\left(\theta^{n}\right)\right)(v(f C) / v(C))$ by (7). Thus if $\xi=\left(\xi_{n}\right) \in \Sigma^{*}$ where $\xi_{n}$ is an $n$-cylinder and $J_{v^{\prime}, v}(\xi)=\lim _{n \rightarrow \infty} v^{\prime}\left(\xi_{n}\right) / v\left(\xi_{n}\right)$, the limit is achieved exponentially fast and $J_{v^{\prime}, v}$ is Hölder continuous on $\Sigma^{*}$. Also, since

$$
\frac{J_{v^{\prime}, v}\left(f_{*} \xi\right)}{J_{v^{\prime}, v}(\xi)} \in\left(1 \pm \mathcal{O}\left(\theta^{n}\right)\right) \cdot \frac{v^{\prime}\left(f \xi_{n}\right)}{v^{\prime}\left(\xi_{n}\right)} \cdot \frac{v\left(\xi_{n}\right)}{v\left(f \xi_{n}\right)} \in 1 \pm \mathcal{O}\left(\theta^{n}\right),
$$

$J_{v^{\prime}, v}\left(f_{*} \xi\right)=J_{v^{\prime}, v}(\xi)$, i.e. $J_{v^{\prime}, v}$ is $f_{*}$-invariant. Therefore, it is constant on a dense set of $\Sigma^{*}$, for example the full backward orbit of a single point. Since it is Hölder continuous it must be constant everywhere and therefore equal to one everywhere. Thus $v=v^{\prime}$ and $v$ is the unique measure satisfying (7). It follows that $v=\lim _{s \backslash P} v_{s}$. A similar argument shows that $v^{*}$ is the unique measure satisfying (8) and $v^{*}=\lim _{s \backslash P} v_{s}^{*}$.

By the properties of the weight function $\ell$ and by (7) for all $n$-cylinders $C$ we get

$$
\frac{v(C)}{\ell(C) e^{-n P}}=\frac{v\left(f^{n} C\right)}{\ell\left(f^{n} C\right)} \cdot \prod_{j=0}^{n-1} \frac{v\left(f^{j} C\right)}{v\left(f^{j+1} C\right) e^{-P}} \cdot \frac{\ell\left(f^{j+1} C\right)}{\ell\left(f^{j} C\right)} \in \frac{v\left(f^{n} C\right)}{\ell\left(f^{n} C\right)} \prod_{j=0}^{n-1}\left(1 \pm \mathcal{O}\left(\theta^{j}\right)\right) .
$$

Thus, the ratios $v(C) / \ell(C) e^{-n P}$ are uniformly bounded away from 0 and $\infty$. Similarly as above, using (8) instead of (7), we obtain that the ratios $v^{*}\left(C^{*}\right) / \ell(C) e^{-n P}$ are uniformly bounded away from 0 and $\infty$. Therefore,

$$
\lim _{s \searrow P} \sum_{C} \ell(C) e^{-n(C) s} \geq c_{1} \lim _{s \searrow P} \sum_{C} v(C) e^{-n(C)(P-s)} \geq c_{2} \lim _{s \searrow P} \sum_{n=1}^{\infty} e^{-n(P-s)}
$$

diverges at $s=P$. The first sum is over all cylinders $C$. 
Therefore, since $v$ and $v *$ are the unique probability measures satisfying respectively (7) and (8), we deduce that $v=\lim _{s \searrow P} \rho_{s}$ and $\nu^{*}=\lim _{s \searrow P} \rho_{s}^{*}$ where $\rho_{s}$ and $\rho_{s}^{*}$ are defined as $v_{s}$ and $v_{s}^{*}$ above, but with $k \equiv 1$. For all cylinders $C$ and $D$, it follows that

$$
\frac{v(C)}{v(D)}=\lim _{s \searrow P} \frac{\sum_{C^{\prime} \subset C} \ell\left(C^{\prime}\right) e^{-n\left(C^{\prime}\right) s}}{\sum_{D^{\prime} \subset D} \ell\left(D^{\prime}\right) e^{-n\left(D^{\prime}\right) s}}=r_{\ell}(C: D),
$$

which ends the proof of the first assertion of this theorem.

From now on in this proof we assume that the weight function $\ell$ matches. In this case $\sum_{C_{n} \subset C} \ell\left(C_{n}\right) / \sum_{D_{n-1} \subset C} \ell\left(D_{n-1}\right) \in\left(1 \pm \mathcal{O}\left(\theta^{n}\right)\right)$ if the first and second sums are, respectively, over all $n$-cylinders and all $(n-1)$-cylinders contained in $C$. Thus $\sum_{C_{n}} \ell\left(C_{n}\right)=\mathcal{O}(1)$ and consequently $\sum_{C} \ell(C) e^{-n(C) s}=\mathcal{O}\left(\sum_{n=0}^{\infty} e^{-n s}\right)$ converges for every $s>0$ and diverges at $s=0$. This implies that $P=0$. Furthermore, we obtain that

$$
r_{\ell}(C: D) \in\left(1 \pm \mathcal{O}\left(\theta^{n}\right)\right) \frac{\ell(C)}{\ell(D)}
$$

where $C$ and $D$ are contained in a common $n$-cylinder. This implies (4).

For all cylinder $\Sigma_{w}$, we have the fact that

$$
\frac{\nu^{*}\left(f_{*}^{-1} \Sigma_{w}^{*}\right)}{\nu^{*}\left(\Sigma_{w}^{*}\right)} \approx \frac{\rho_{s}^{*}\left(f_{*}^{-1} \Sigma_{w}^{*}\right)}{\rho_{s}^{*}\left(\Sigma_{w}^{*}\right)}=\frac{\sum_{m D=C: f^{k} C=\Sigma_{w}} \ell(D) e^{-n(D) s}}{\sum_{f^{k} C=\Sigma_{w}} \ell(C) e^{-n(C) s}}
$$

with the approximation converging as $s \searrow 0$. Since the ratios $\ell(C) / \ell(m C)$ converge exponentially fast along backward orbits there are continuous functions $\tau_{1}(s)$ and $\tau_{2}(s)$ which converge to one as $s \searrow 0$ such that for all cylinders $\Sigma_{w}$,

$$
\tau_{1}(s)<\frac{\sum_{m D=C: f^{k} C=\Sigma_{w}} \ell(D) e^{-n(D) s}}{\sum_{f^{k} C=\Sigma_{w}} \ell(C) e^{-n(C) s}}<\tau_{2}(s) .
$$

Thus we deduce that $v^{*}\left(f_{*}^{-1} C^{*}\right)=v^{*}\left(C^{*}\right)$ for all cylinders and hence the fact that $v^{*}$ is $f_{*}$-invariant. It follows from this that if we define $\mu$ on $\Sigma$ by $\mu(C)=v^{*}\left(C^{*}\right)$ for all cylinders $C$ of $\Sigma$ then $\mu$ is a $f$-invariant probability measure on $\Sigma$. The fact that it is a measure follows from the $f_{*}$-invariance of $v^{*}$ and the fact that it is $f$-invariant follows from the fact that $v^{*}$ is a probability measure.

Now we consider the ratios $\mu\left(C_{1}\right) / \mu\left(C_{2}\right)=\nu^{*}\left(C_{1}^{*}\right) / \nu^{*}\left(C_{2}^{*}\right)$ where $C_{1}$ and $C_{2}$ are cylinders and $C_{1}$ is contained in $C_{2}$. Then there exists $r \geq 0$ such that $m^{r} C_{1}=C_{2}$. In this case, $f_{*}^{r} C_{1}^{*}=C_{2}^{*}$. Thus the ratio is approximated by

$$
\frac{\sum_{m_{*}^{k} C^{*}=C_{1}^{*}} \ell(C) e^{-n(C) s}}{\sum_{m_{*}^{k} C^{*}=f_{*}^{r} C_{1}^{*}} \ell(C) e^{-n(C) s}}=\frac{\sum_{f^{k} C=C_{1}} \ell(C) e^{-n(C) s}}{\sum_{f^{k} C=m^{r} C_{1}} \ell(C) e^{-n(C) s}}
$$

with convergence as $s \searrow 0$. To each summand $\ell(C)$ of the top sum there corresponds a summand $\ell\left(C^{\prime}\right)$ of the bottom sum such that $m^{r} C=C^{\prime}$, and the pair $\left(C, C^{\prime}\right)$ is mapped by some power of $f$ onto the pair $\left(C_{1}, C_{2}\right)$. It follows that $\ell(C) / \ell\left(C^{\prime}\right) \in$ $\left(1 \pm \mathcal{O}\left(\theta^{n\left(C_{2}\right)}\right)\right) \ell\left(C_{1}\right) / \ell\left(C_{2}\right)$ where the constant of proportionality in the $\mathcal{O}$ term is independent of $C, C^{\prime}, C_{1}$ and $C_{2}$. Thus we deduce that the last term for $s=0$ of (12) is in the interval $\left(1 \pm \mathcal{O}\left(\theta^{n}\right)\right) \ell\left(C_{1}\right) / \ell\left(C_{2}\right)$. We have proved that if $C_{2}$ is an $n$-cylinder then

$$
\frac{\mu\left(C_{1}\right)}{\mu\left(C_{2}\right)} \in\left(1 \pm \mathcal{O}\left(\theta^{n}\right)\right) \frac{\ell\left(C_{1}\right)}{\ell\left(C_{2}\right)}
$$


Theorem 1(i) and (ii) follow from this.

It remains to prove Theorem 1(iii), the ratio decomposition. To do this recall the meaning of $C\left(C_{n}\right)$ given in $\S 2.3$. If $C_{p}$ is a primary cylinder let $\mathcal{C}_{n}\left(C_{p}\right)$ denote the set of $n$-cylinders $C$ such that $f^{n-1} C=C_{p}$. Let $C_{p}$ be the primary cylinder containing $\Sigma_{w}$. We have

$$
\begin{aligned}
\mu\left(\Sigma_{w}\right) & \stackrel{1}{=} \sum_{C_{n} \in \mathcal{C}_{n}\left(C_{p}\right)} \frac{\mu\left(\Sigma_{w}\left(C_{n}\right)\right)}{\mu\left(C_{n}\right)} v^{*}\left(C_{n}^{*}\right) \\
& \stackrel{2}{\approx} \sum_{C_{n} \in \mathcal{C}_{n}\left(C_{p}\right)} \frac{\ell\left(\Sigma_{w}\left(C_{n}\right)\right)}{\ell\left(C_{n}\right)} v^{*}\left(C_{n}^{*}\right) \rightarrow \int_{C_{p}^{*}} r_{\ell, \xi}\left(\Sigma_{w}\right) v^{*}(d \xi)
\end{aligned}
$$

as $n \rightarrow \infty$. The equality marked $\stackrel{1}{=}$ follows from the $f$-invariance of $\mu$ and also by duality, that marked $\stackrel{2}{\approx}$ from (13) and the convergence from property (ii) of the potential, from the definition of $r_{\xi}\left(\Sigma_{w}\right)$ in $\S 2.3$ and the comments in Remark 1 .

The final point is to check uniqueness of invariant measures satisfying either (i), (ii) or (iii). Since (i) implies (ii) it suffices to check (ii) to verify both. However, if $\rho^{*}$ is another measure satisfying the condition in part (ii) then one can prove that $\rho=\mu$ in a similar fashion to the proof of the uniqueness of $v$ above, using $\rho^{*}$ and $v^{*}$, the fact that $f_{*} C^{*}=(m C)^{*}$, and condition (ii) of this theorem.

Suppose that $\rho$ is a measure satisfying the ratio decomposition (iii) of the theorem and let $\rho^{*}$ denote its dual. First, we note that if $\xi_{n+1}$ is an $n+1$-cylinder and $\xi_{n}=f \xi_{n+1}$ then $r_{\ell}\left(C\left(\xi_{n+1}\right): \xi_{n+1}\right)=\left(1 \pm \mathcal{O}\left(\theta^{n}\right)\right) r_{\ell}\left(C\left(\xi_{n}\right): \xi_{n}\right)$. Moreover, since $\rho$ is $f$-invariant, $\sum_{\xi_{n+1}^{*}} \rho^{*}\left(\xi_{n+1}^{*}\right)=\rho^{*}\left(\xi_{n}^{*}\right)$ where the sum is over all $\xi_{n+1}^{*}$ contained in $\xi_{n}^{*}$ or equivalently over all $f$-preimages $\xi_{n+1}$ of $\xi_{n}$. Thus

$$
\sum_{(n+1) \text {-cyls. } \xi_{n+1}} r_{\ell}\left(C\left(\xi_{n+1}\right): \xi_{n+1}\right) \rho^{*}\left(\xi_{n+1}\right)=\left(1 \pm \mathcal{O}\left(\theta^{n}\right)\right) \sum_{n \text {-cyls. } \xi_{n}} r_{\ell}\left(C\left(\xi_{n}\right): \xi_{n}\right) \rho^{*}\left(\xi_{n}\right) \text {. }
$$

This with condition (iii) proves Remark 3.

Therefore, if $C$ and $D$ are cylinders of $\Sigma$ contained in the cylinder $E$ and $\xi \in \Sigma^{*}$ has $E \subset \xi_{0}$ then, denoting by $\mathcal{C}_{n}(E)$ the set of $n$-cylinders $C^{\prime}$ such that $f^{n-1} C^{\prime}$ contains $E$,

$$
\begin{aligned}
\frac{\rho(C)}{\rho(D)} & \approx \frac{\sum_{\xi_{n} \in \mathcal{C}_{n}(E)} r_{\ell}\left(C\left(\xi_{n}\right): \xi_{n}\right) \rho^{*}\left(\xi_{n}\right)}{\sum_{\xi_{n} \in \mathcal{C}_{n}(E)} r_{\ell}\left(D\left(\xi_{n}\right): \xi_{n}\right) \rho^{*}\left(\xi_{n}\right)} \\
& =\frac{\sum_{\xi_{n} \in \mathcal{C}_{n}(E)} r_{\ell}\left(C\left(\xi_{n}\right): D\left(\xi_{n}\right)\right) r_{\ell}\left(D\left(\xi_{n}\right): \xi_{n}\right) \rho^{*}\left(\xi_{n}\right)}{\sum_{\xi_{n} \in \mathcal{C}_{n}(E)} r_{\ell}\left(D\left(\xi_{n}\right): \xi_{n}\right) \rho^{*}\left(\xi_{n}\right)} \\
& \in\left(1 \pm \mathcal{O}\left(\theta^{n}\right)\right) \frac{\ell(C)}{\ell(D)}
\end{aligned}
$$

because

$$
r_{\ell}\left(C\left(\xi_{n}\right): D\left(\xi_{n}\right)\right)=\frac{\ell\left(C\left(\xi_{n}\right)\right)}{\ell\left(D\left(\xi_{n}\right)\right)} \in\left(1 \pm \mathcal{O}\left(\theta^{n}\right)\right) \frac{\ell(C)}{\ell(D)}
$$

since $C$ and $D$ are in the $n$-cylinder $E$. Thus if $\xi=\left(\xi_{n}\right)_{n=0}^{\infty} \in \Sigma^{*}$ then

$$
\frac{\rho\left(\xi_{n}\right)}{\rho\left(m \xi_{n}\right)} \in\left(1 \pm \mathcal{O}\left(\theta^{n}\right)\right) \sigma_{\ell}(\xi)
$$

by (14) and, consequently, $\rho$, like $\mu$, satisfies condition (ii) of the theorem. But we have already shown that there is only one measure satisfying this. Hence $\rho=\mu$. 
Proof of Corollary 1. First, we apply Theorem 1 to the weight system $\ell$ to obtain the measure $v$. Then we consider the new weight system $\ell_{v}(C)=v(C)$. By Theorem 1 this is Hölder and clearly, since $v$ is a probability measure, it satisfies the matching condition. Now apply Theorem 1 to this to obtain measures $v_{1}, v_{1}^{*}$ and $\mu=\mu_{1}$ (corresponding to $v$, $\nu^{*}$ and $\mu$ of the theorem). It follows immediately from Theorem 1 that $\mu$ is the required Gibbs state. As is well known, since $\mu$ has a Hölder Jacobian it is ergodic. Therefore, it is the unique invariant measure in its measure class and hence the unique invariant measure for which the ratios $\mu(C) / \ell(C) e^{-n(C) P}$ are uniformly bounded away from 0 and $\infty$.

Acknowledgements. We thank the Gulbenkian Foundation, FCT, PRAXIS, ESFPRODYN and the Centro de Matemática Aplicada da Universidade do Porto for their financial support of A. A. Pinto and the UK Science and Engineering Research Council and the EU for their financial support of D. A. Rand.

\section{REFERENCES}

[1] R. Bowen. Equilibrium States and the Ergodic Theory of Axiom A Diffeomorphisms (Lecture Notes in Mathematics, 470). Springer, New York, 1975.

[2] S. J. Paterson. The limit set of a Fuchsian group. Acta. Math. 136 (1976), 241-273.

[3] A. A. Pinto and D. A. Rand. Geometric measures for hyperbolic surface dynamics. Preprint, 1999.

[4] D. Sullivan. Conformal Dynamical Systems (Springer Lecture Notes in Mathematics, 1007). Springer, New York, 1983, pp. 725-752. 